\title{
Evolution of massive black holes
}

\author{
By Marta Volonteri ${ }^{1}$ \\ ${ }^{1}$ University of Michigan, Ann Arbor, MI 48109, USA
}

\begin{abstract}
Supermassive black holes are nowadays believed to reside in most local galaxies. Accretion of gas and black hole mergers play a fundamental role in determining the two parameters defining a black hole: mass and spin. I briefly review here some of the physical processes that are conducive to the evolution of the massive black hole population. I'll discuss black hole formation processes that are likely to place at early cosmic epochs, and how massive black hole evolve in a hierarchical Universe. The mass of the black holes that we detect today in nearby galaxy has mostly been accumulated by accretion of gas. While black hole-black hole mergers do not contribute substantially to the final mass of massive black holes, they influence the occupancy of galaxy centers by black hole, owing to the chance of merging black holes being kicked from their dwellings due to the "gravitational recoil". Similarly, accretion leaves a deeper imprint on the distribution of black hole spins than black hole mergers do. The differences in accretion histories for black holes hosted in elliptical or disc galaxies may reflect on different spin distributions.
\end{abstract}

\section{Introduction}

Black holes (BHs), as physical entities, span the full range of masses, from tiny BHs predicted by string theory, to monsters weighting by themselves almost as much as a dwarf galaxy (massive black holes, MBHs). Notwithstanding the several orders of magnitude difference between the smallest and the largest $\mathrm{BH}$ known, we believe that all of them can be described by only three parameters: mass, spin and charge. Astrophysical BHs are even simpler system, as charge can be neglected as well. The interaction between astrophysical BHs and their environment is where complexity enters the game. I will focus here on the formation and evolution of MBHs, with masses above thousands solar masses, and how we believe their evolution is symbiotic with that of their host.

Let's start by recalling that MBHs in galaxy centers are far from being really "black": we can easily trace the presence, as they are the engines powering the luminous quasars that have been detected up to high redshift. Nowadays we can detect in neighboring galaxies the dead remnants of this bright past activity. It is indeed well established that the centers of most local galaxies host MBHs with masses in the range $M_{B H} \sim 10^{6}-$ $10^{9} M_{\odot}$ (e.g., Ferrarese \& Merritt 2000; Kormendy \& Gebhardt 2001; Richstone et al. 1998). The MBH population may extend down to the smallest masses. For example, the dwarf Seyfert 1 galaxy POX 52 is thought to contain a BH of mass $M_{B H} \sim 10^{5} M_{\odot}$ (Barth et al. 2004). At the other end, however, the Sloan Digital Sky survey detected luminous quasars at very high redshift, $z>6$. Follow-up observations confirmed that at least some of these quasars are powered by supermassive black holes with masses $\simeq 10^{9} M_{\odot}$ (Barth et al. 2003; Willott et al. 2005). We are therefore left with the task of explaining the presence of very big MBHs when the Universe is less than 1 Gyr old, and of much smaller BHs lurking in 13 Gyr old galaxies.

\section{Massive black holes in a hierarchical Universe}

The demography of massive black holes in the local Universe has been clarified in the last ten years by studies of the central regions of relatively nearby galaxies (mainly with quiescent nuclei). The mass of MBHs detected in neighboring galaxies scales with the bulge luminosity - or stellar velocity dispersion - of their host galaxy (Ferrarese \& Merritt 
2000; Gebhardt et al. 2000; Tremaine et al. 2002), suggesting a single mechanism for assembling black holes and forming spheroids in galaxy halos. The evidence is therefore in favour of a co-evolution between galaxies, black holes and quasars. In the currently favoured cold dark matter cosmogonies (Spergel et al. 2007), present-day galaxies have been assembled via a series of mergers, from small-mass building blocks which form at early cosmic times. In this paradigm galaxies experience multiple mergers during their lifetime. If most galaxies host BHs in their centre, and a local galaxy has been made up by multiple mergers, then a black hole binary is a natural evolutionary stage. During each galaxy merger event, the central black holes already present in each galaxy would be dragged to the centre of the newly formed galaxy via dynamical friction $(\approx 0.1-10 \mathrm{pc})$, and then if/when they get close $(\approx 0.01-0.001 \mathrm{pc})$ the black hole binary would coalesce via emission of gravitational radiation. The gap between where dynamical friction ceases to be efficient $\left(a_{h} \simeq G m_{2} /\left(4 \sigma_{*}^{2}\right)\right)$ and where emission of gravitational waves takes over, at binary separations about two orders of magnitude smaller, could be the bottleneck of the merger process.

In gas-poor systems, the sub-parsec evolution of the binary while gravitational radiation emission is still negligible, may be largely determined by three-body interactions with background stars (Begelman et al. 1980), by capturing the stars that pass within a distance of the order of the binary semi-major axis and ejecting them at much higher velocities (Quinlan 1996; Milosavliević \& Merritt 2001; Sesana et al. 2007a). Dark matter particles will be ejected by decaying binaries in the same way as the stars, i.e. through the gravitational slingshot. The hardening of the binary modifies the density profile, removing mass interior to the binary orbit, depleting the galaxy core of stars and dark matter, and slowing down further decay. We can use a toy model to understand the typical timescales (Volonteri et al. 2003). If we assume that the stellar mass removal scours a core of radius $r_{c}$ and constant density $\rho_{c} \equiv \rho_{*}\left(r_{c}\right)$ into a pre-existing isothermal sphere, the total mass ejected as the binary shrinks from $a_{h}$ to $a<a_{h}$ can be written as

$$
\mathcal{M}_{\mathrm{ej}}=\frac{4}{3} \frac{\sigma_{*}^{2}\left(r_{c}\right)}{G},
$$

where $m_{2}$ is the least massive $\mathrm{BH}$ in the binary, and $\sigma_{*}$ is the velocity dispersion of the stellar system. The core radius then grows as

$$
r_{c}(t) \approx \frac{3}{4 \sigma_{*}^{2}} G\left(m_{1}+m_{2}\right) \int_{a(t)}^{a_{h}} \frac{1}{a} d a .
$$

The binary separation quickly falls below $r_{c}$ and subsequent evolution is slowed down due to the declining stellar density, with a hardening time $t_{h}=|a / \dot{a}|=2 \pi r_{c}(t)^{2} /\left(H \sigma_{*} a\right)$ that becomes increasingly long as the binary shrinks and $r_{c}(t)$ increases.

In gas rich systems, however, the orbital evolution of the central MBH is likely dominated by dynamical friction against the surrounding gaseous medium. The available simulations (Escala et al. 2004; Dotti et al. 2006; Maver et al. 2006) show that the binary can shrink to about parsec or slightly subparsec scale by dynamical friction against the gas, depending on the gas thermodynamics. The interaction between a $\mathrm{BH}$ binary and an accretion disc can also lead to a very efficient transport of angular momentum, and drive the secondary $\mathrm{BH}$ to the regime where emission of gravitational radiation dominates on short timescales, comparable to the viscous timescale (Armitage \& Natarajan 2005; Gould \& Rix 2000). 


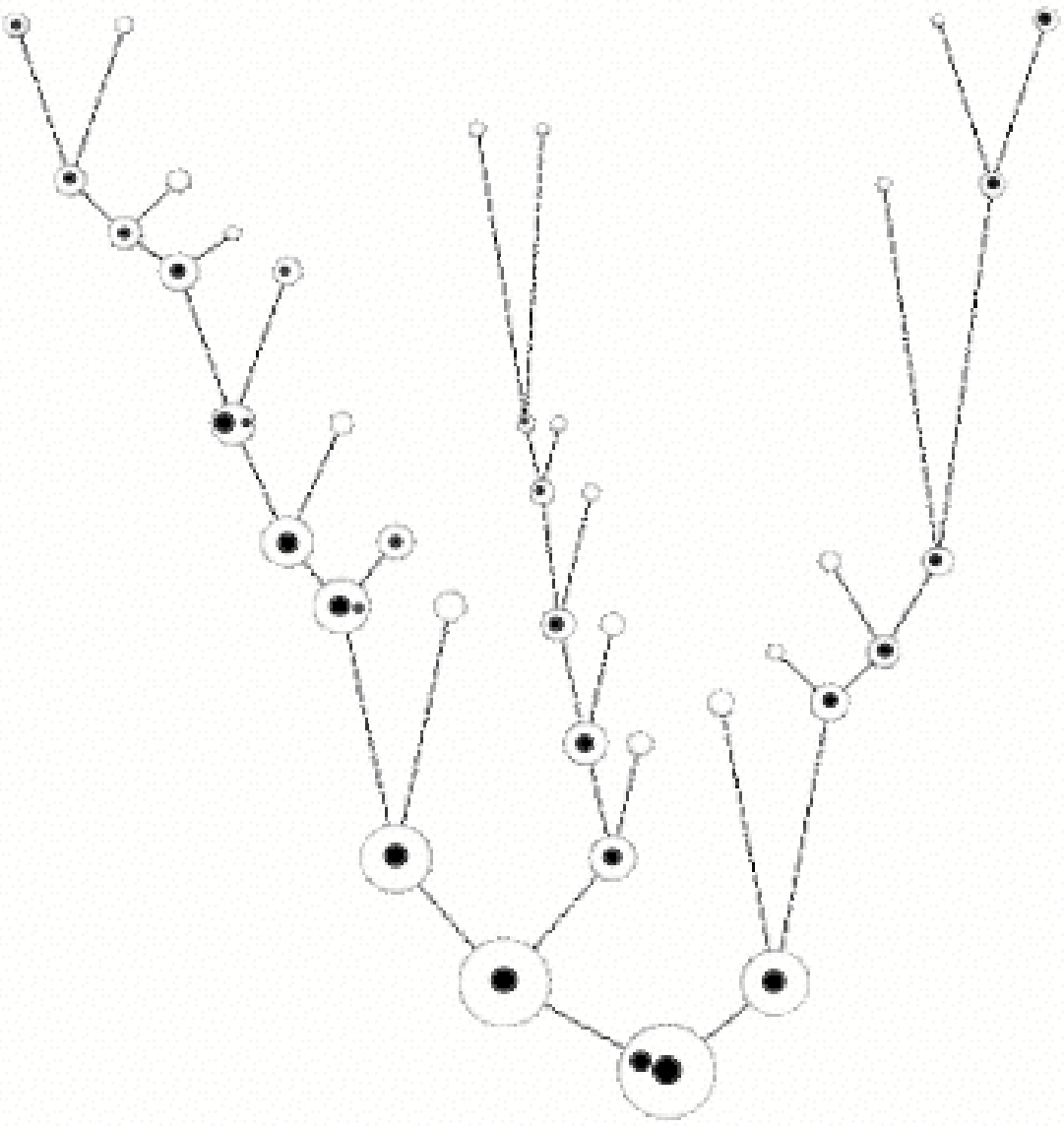

Figure 1. A cartoon of the assembly of a galaxy and its central black hole in cold-dark matter cosmology. Time increases from top to bottom, and every junction between two "branches" of the merger tree marks a galaxy merger. The central MBHs are indicated by black dots (not in scale). In this case the final galaxy is assembled from the merger of twenty smaller galaxies, containing a total of four seed black holes, resulting in four mergers of binary black holes.

The viscous timescale depends on the properties of the accretion disc and of the binary:

$$
t_{\mathrm{vis}}=0.1 \operatorname{Gyr} a_{\mathrm{pc}}^{3 / 2}\left(\frac{H}{R}\right)_{0.1}^{-2} \alpha_{0.1}^{-1}\left(\frac{m_{1}}{10^{4} M_{\odot}}\right)^{-1 / 2}
$$

where $a_{\mathrm{pc}}$ is the initial separation of the binary when the secondary MBH starts interacting with the accretion disc in units of parsec, $(h / r)$ is the aspect ratio of the accretion disc, $h / r=0.1$ above, $\alpha$ is the Shakura \& Sunyaev viscosity parameter, $\alpha=0.1$ above, and $m_{1}$ is the mass of the primary $\mathrm{MBH}$, in solar masses. The emission of gravitational waves takes over the viscous timescales at a separation (Armitage \& Natarajan 2005):

$$
a_{\mathrm{GW}}=10^{-8} \mathrm{pc}\left(\frac{\mathrm{H}}{\mathrm{R}}\right)_{0.1}^{-16 / 5} \alpha_{0.1}^{-8 / 5} \mathrm{q}_{0.1}^{3 / 5}\left(\frac{\mathrm{m}_{1}}{10^{4} \mathrm{M}_{\odot}}\right)
$$




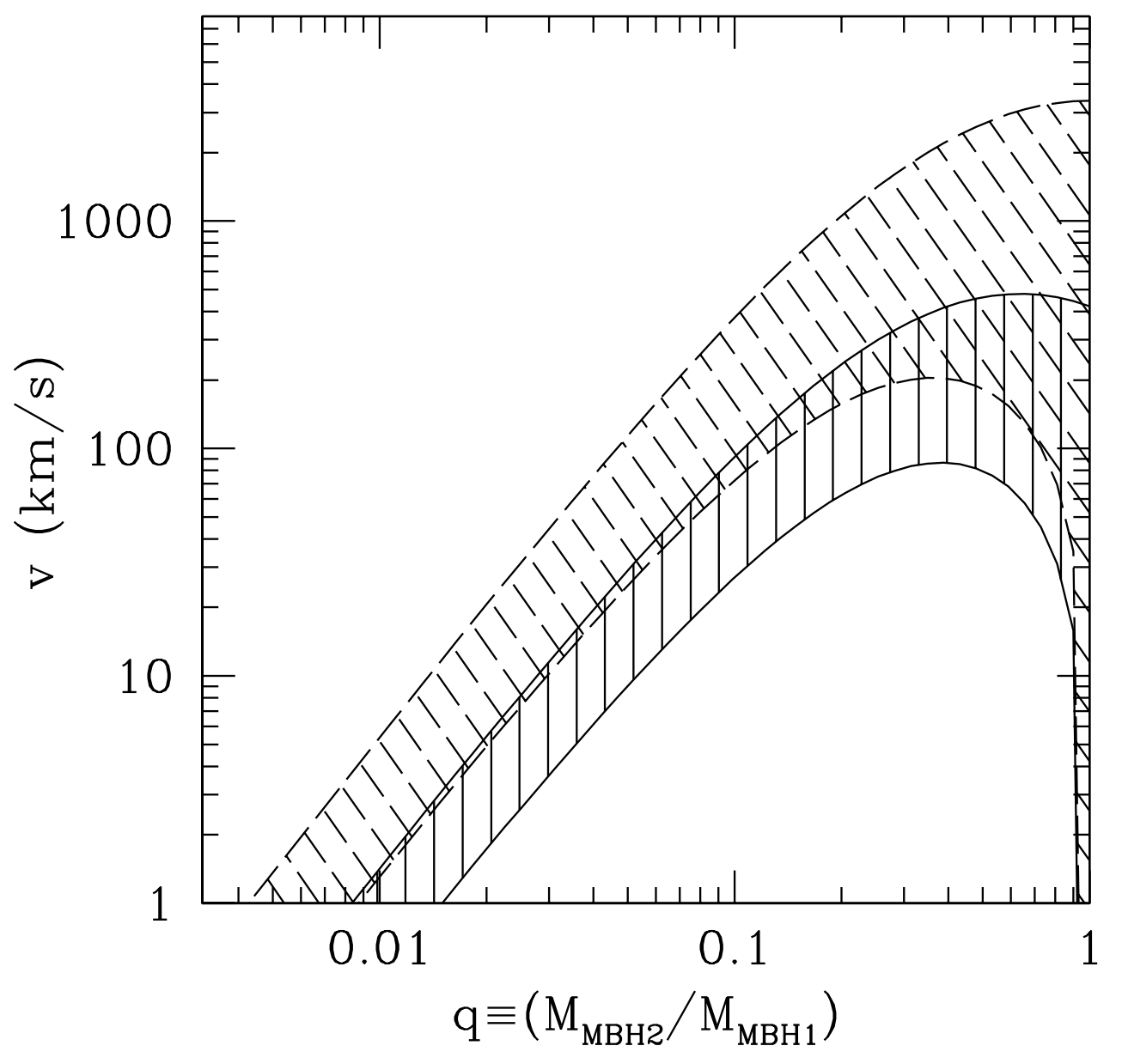

Figure 2. Recoil velocity of spinning black holes as a function of binary mass ratio, q. Solid curves: spin axis aligned (or anti-aligned) with the orbital angular momentum (Baker et al. 2007). Dashed curves: spin axis in the orbital plane (Campanelli et al. 2007). For every mass ratio we plot the combination of spins, $\hat{a}_{1}$ and $\hat{a}_{2}$ which minimizes (lower curves) or maximizes (upper curves) the recoil velocity. From Volonteri (2007)

where $q=m_{2} / m_{1} \lesssim 1$, is the binary mass-ratio. The timescale for coalescence by emission of gravitational waves from $a_{\mathrm{GW}}$ is much shorter than the Hubble time:

$$
t_{\mathrm{gr}}=2.3 \times 10^{6} \mathrm{yr}\left(\frac{a(t)}{0.1 \mathrm{pc}}\right)^{4}\left(\frac{m_{1} m_{2}(m 1+m 2)}{2 \times 10^{18} \mathrm{M}_{\odot}}\right) .
$$

Hence, the physical processes driving the evolution of $\mathrm{MBH}$ binaries are likely redshift and environmental dependent. Fast mergers are probably common in high redshift young galaxies, and sluggish binaries might reach an impasse in low-redshift gas poor spheroids.

Somehow counter-intuitively fast MBH mergers at very high redshift can bring an overall damage to the growth of the $\mathrm{MBH}$ population, rather than contribute to the build-up of more massive holes. This is due to the so-called "gravitational recoil" (a.k.a. rocket, kick). When the members of a black hole binary coalesce, the center of mass of 
the coalescing system recoils due to the non-zero net linear momentum carried away by gravitational waves in the coalescence. This recoil could be so violent that the merged hole breaks loose from shallow potential wells, especially in small mass pregalactic building blocks (Figure 2).

Comparing the recoil velocity to the escape velocity from their hosts, Volonteri (2007) find that the fraction of "lost" binaries is very high $(>50-90 \%)$ at $z>10$, but it decreases at later times due to a combination of (i) the mass ratio distribution becoming shallower, and, (ii) the hierarchical growth of the hosts (Fig. 3). Schnittman (2007) shows in a very elegant way that, even for large recoils, the very hierarchical nature of structure evolution ensures that a substantial fraction of galaxies retain their BHs, if evolution proceeds over a long series of mergers (see also Menou et al. 2001). As, especially at high redshift, binaries represent the exception, rather than the rule, the possible ejection of most binaries before $z \simeq 5$ is not a threat to the evolution of the $\mathrm{MBH}$ population that has been detected in nearby galaxies.

Although the gravitational recoil does not damage to the evolution of the MBH population that we observe locally, it can be dangerous in very special cases. Haiman (2004) pointed out that the recoil can be indeed threatening the growth of the MBHs that are believed to be powering the luminous quasars at $z \simeq 6$ detected in the Sloan survey (e.g., Fan et al. 2001). In fact, in such a biased volume, the density of halos where MBH formation can be efficient (either by direct collapse, or via PopIII stars) is highly enhanced. The net result is an higher fraction of binary systems, and binarity is especially common for the central galaxy of the main halo. While the "average" MBH experiences at most one merger in its lifetime, a $\mathrm{MBH}$ hosted in a rare exceptionally massive halo can experience up to a few tens mergers, and the probability of ejecting the central $\mathrm{MBH}$, halting its growth, is $50-80 \%$ at $z>6$ (Volonteri \& Rees 2006). This implies that MBHs at high redshift did not mainly grow via mergers.

\section{Scenarios for massive black hole formation}

A single big galaxy can be traced back to the stage when it was split up in hundreds of smaller components with individual internal velocity dispersions as low as $20 \mathrm{~km} \mathrm{~s}^{-1}$. Did black holes form with the same efficiency in small galaxies (with shallow potential wells), or did their formation had to await the buildup of substantial galaxies with deeper potential wells?

The formation of massive black holes is far less understood than that of their light, stellar mass, counterparts. The "flowing chart" presented by Rees (1978) still stands as a guideline for the possible paths leading to formation of massive $\mathrm{BH}$ seeds in the center of galactic structures. One first possibility is the direct formation of a $\mathrm{BH}$ from a collapsing gas cloud (Haehnelt \& Rees 1993; Loeb \& Rasio 1994; Eisenstein \& Loeb 1995; Bromm \& Loeb 2003; Koushiappas et al. 2004; Begelman et al. 2006; Lodato \& Natarajan 2006). In the most common situations, rotational support can halt the collapse before densities required for $\mathrm{MBH}$ formation are reached. Halos, and their baryonic cores, possess in fact angular momentum, $J$, believed to be acquired by tidal torques due to interactions with neighboring halos. This can be quantified through the so-called spin parameter, which represents the degree of rotational support available in a gravitational system: $\lambda_{\text {spin }} \equiv J|E|^{1 / 2} / G M_{h}^{5 / 2}$, where $E$ and $M_{h}$ are the total energy and mass of the halo.

Let $f_{\text {gas }}$ be the gas fraction of a proto-galaxy mass, and $f_{d}$ the fraction of the gas which can cool; a mass $M=f_{d} f_{\text {gas }} M_{h}$ would then settle into a rotationally supported disc (Mo et al. 1998; Oh \& Haiman 2002) with a scale radius $\simeq \lambda_{\text {spin }} r_{\text {vir }}$, where $r_{\text {vir }}$ is the virial radius of the proto-galaxy. Spin parameters found in numerical simulations are 


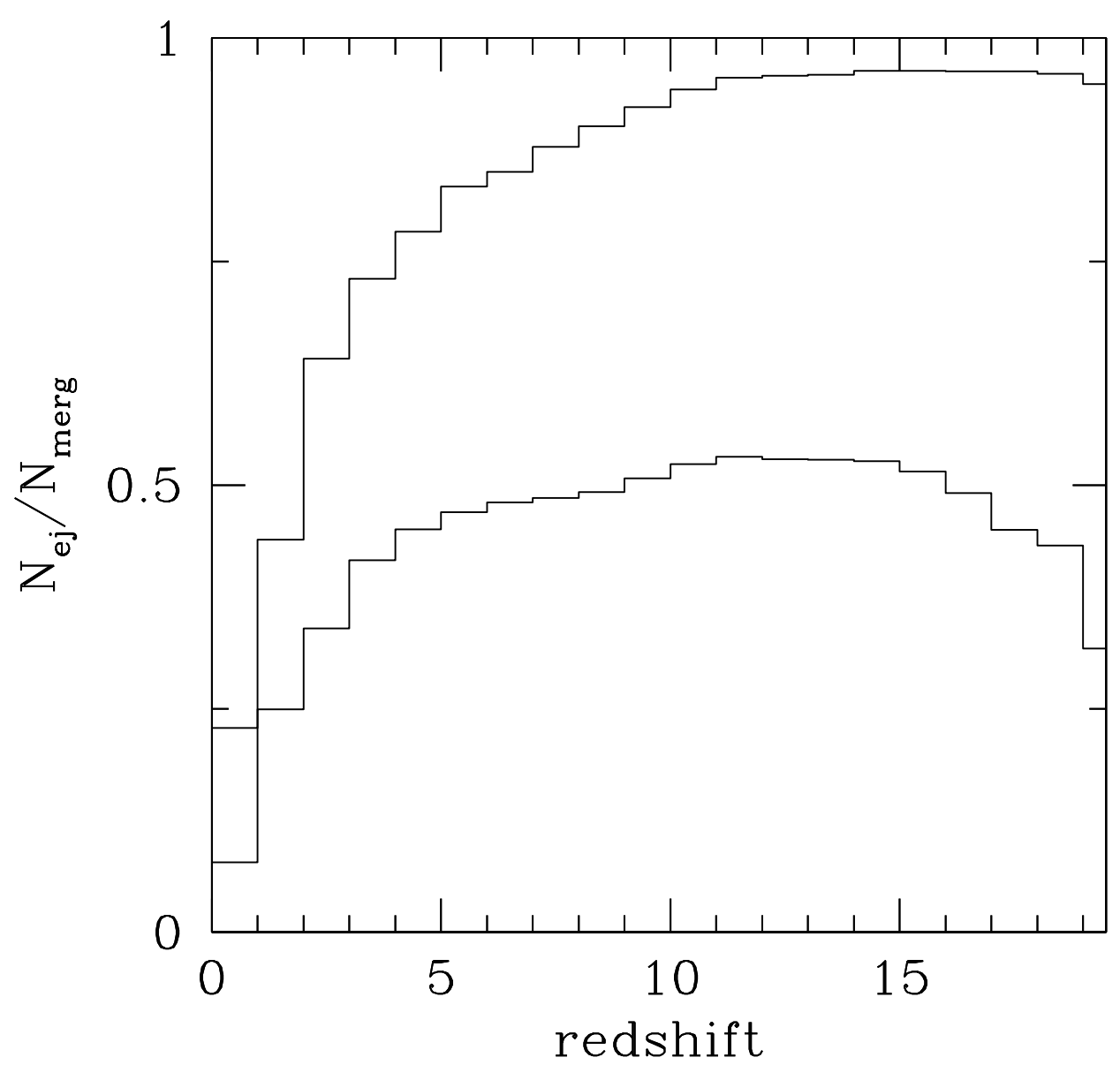

FIGURE 3. Fraction of ejected binaries as a function of redshift for Schwarzschild MBHs (lower histogram) and spinning MBHs (upper histogram), assuming isotropic orbits. A binary is defined ejected if the recoil velocity is larger than the escape velocity from the host.

distributed log-normally in $\lambda_{\text {spin }}$, with mean $\bar{\lambda}_{\text {spin }}=0.04$ and standard deviation $\sigma_{\lambda}=$ 0.5 (e.g., Bullock et al. 2001; van den Bosch et al. 2002). The tidally induced angular momentum would therefore be enough to provide centrifugal support at a distance $\simeq$ $20 \mathrm{pc}$ from the center, and halt collapse. Additional mechanisms inducing transport of angular momentum are needed to further condense the gas.

The loss of angular momentum can be driven either by (turbulent) viscosity or by global dynamical instabilities, such as the "bars-within-bars" mechanism (Shlosman et al. 1989; Begelman et al. 2006). The gas can therefore condense to form a central massive object, either a supermassive star, which eventually becomes subject to post-Newtonian gravitational instability and forms a seed $\mathrm{BH}$, or via a low-entropy star-like configuration where a small black hole forms in the core and grows by accreting the surrounding envelope (Begelman, Rossi \& Armitage 2007). The masses of the seeds predicted by different models vary, but they are typically in the range $M_{B H} \sim 10^{4}-10^{6} M_{\odot}$. 


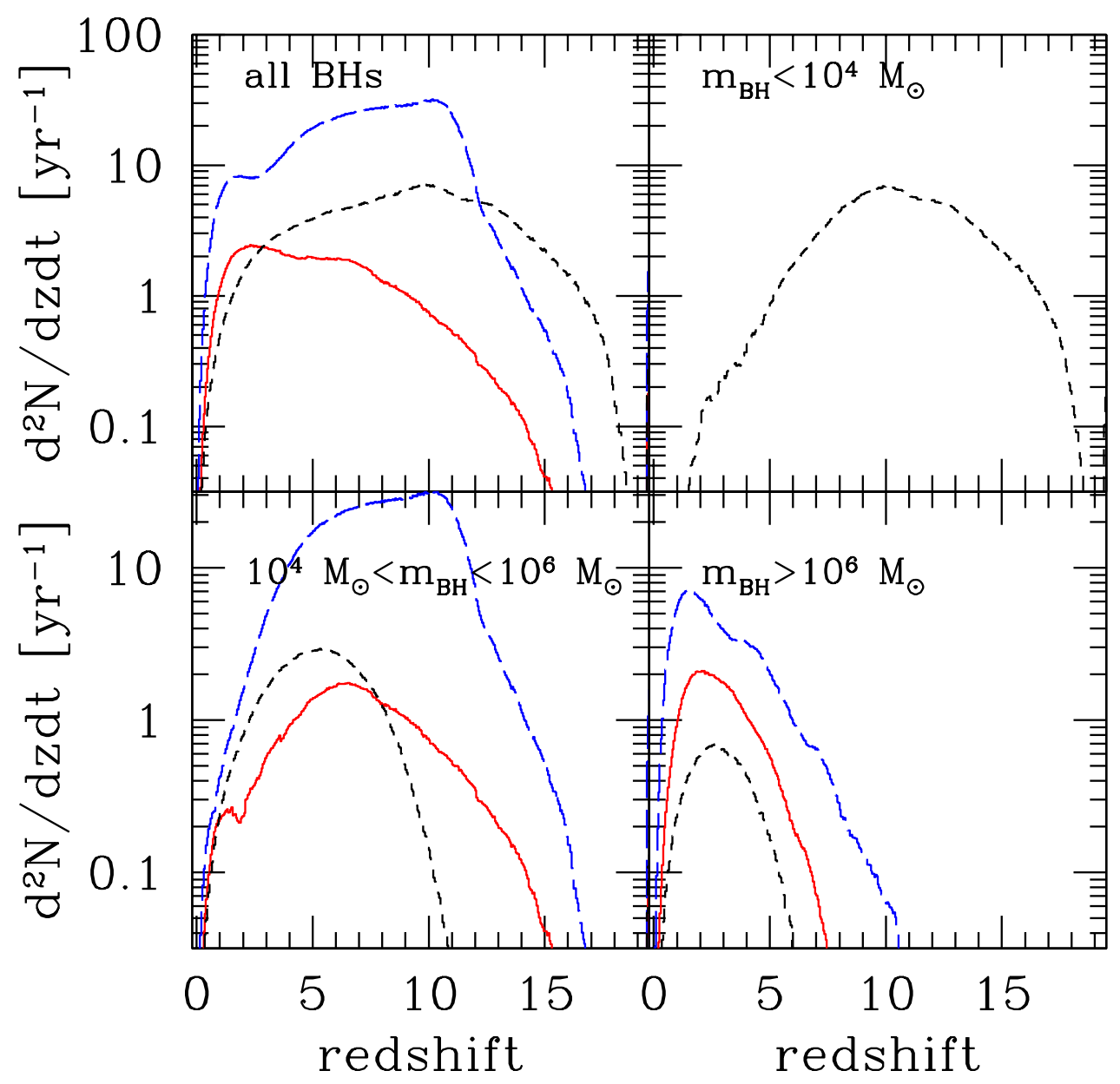

Figure 4. Predicted rate of $\mathrm{MBH}$ binary coalescences per unit redshift, in different $\mathrm{MBH}$ mass intervals. Solid curve: MBH seeds from PopIII stars. Long dashed curve: MBH seeds from direct collapse (Koushiappas et al. 2004). Short dashed curve: MBH seeds from direct collapse (Begelman, Volonteri \& Rees 2006). Adapted from Sesana et al. 2007.

Alternatively, the seeds of MBHs can be associated with the remnants of the first generation of stars, formed out of zero metallicity gas. The first stars are believed to form at $z \gtrsim 10$ in halos which represent high- $\sigma$ peaks of the primordial density field. The main coolant, in absence of metals, is molecular hydrogen, which is a rather inefficient coolant. The inefficient cooling might lead to a very top-heavy initial stellar mass function, and in particular to the production of very massive stars with masses $>100 M_{\odot}($ Carr, Bond, $\&$ Arnett 1984). If very massive stars form above $260 M_{\odot}$, they would rapidly collapse to massive BHs with little mass loss (Frver et al. 2001), i.e., leaving behind seed BHs with masses $M_{B H} \sim 10^{2}-10^{3} M_{\odot}$ (Madau \& Rees 2001; Volonteri et al. 2003).

\subsection{Observational tests of $M B H$ formation scenarios}

What are the possible observational tests of $\mathrm{MBH}$ formation scenarios? Detection of gravitational waves from seeds merging at the redshift of formation (Sesana et al. 2007b) 
is probably one of the best ways to discriminate among formation mechanisms. The planned Laser Interferometer Space Antenna (LISA) in principle is sensible to gravitational waves from binary MBHs with masses in the range $10^{3}-10^{6} M_{\odot}$ basically at any redshift of interest. A large fraction of coalescences will be directly observable by LISA, and on the basis of the detection rate, constraints can be put on the MBH formation process. Different theoretical models for the formation of MBH seeds and dynamical evolution of the binaries predict merger rates that largely vary one from the other (Figure 4. Sesana et al. (2007b)).

The imprint of different formation scenarios can also be sought in observations at lower redshifts (Volonteri, Lodato \& Natarajan 2007). Since during the quasar epoch MBHs increase their mass by a large factor, signatures of the seed formation mechanisms are likely more evident at earlier epochs. Fig. 5 compares the integrated comoving mass density in MBHs to the expectations from Soltan-type arguments (F. Haardt, private communication), assuming that quasars are powered by radiatively efficient flows (for details, see Yu \& Tremaine 2002; Elvis et al. 2002; Marconi et al. 2004). The curves differ only with respect to the $\mathrm{MBH}$ formation scenario. We either assume that seeds are Population III remnants (black curve), or that seeds are formed via direct collapse with different efficiencies (Lodato \& Natarajan 2006). While during and after the quasar epoch the mass densities in our theoretical models differ by less than a factor of 2 , at $z>3$ the differences become more pronounced. The comoving mass density, an integral constraint, is reasonably well determined out to $z=3$ but is still poorly known at higher redshifts. The increasing area and depth of high redshift survey, especially in X-rays, will increase the strength of our constraints (Salvaterra et al. 2007).

In our neighbourhood, the best diagnostic of $\mathrm{MBH}$ formation mechanisms would be the measure of $\mathrm{MBH}$ masses in low-luminosity galaxies. This can be understood in terms of the cosmological bias. The progenitors of massive galaxies (or clusters of galaxies) have both a high probability of hosting MBH seeds (cfr. Madau \& Rees 2001), and a high probability that the central MBH is not "pristine", that is it has increased its mass by accretion, or it has experienced mergers and dynamical interactions. In the case of low-bias systems, such as isolated dwarf galaxies, very few of the high- $z$ progenitors have the deep potential wells needed for gas retention and cooling, a prerequisite for $\mathrm{MBH}$ formation. The signature of the efficiency of the formation of MBH seeds will consequently be stronger in isolated dwarf galaxies. Hence, $\mathrm{MBH}$ formation models are distinguishable at the low mass end of the $\mathrm{BH}$ mass function, while at the high mass end the effect of initial seeds appears to be sub-dominant. The clearest signature of massive seeds, compared to Population III remnants, would be a lower limit of order the typical mass of seeds to the mass of MBHs in galaxy centers, as shown in Fig. 6. Additionally, the fraction of galaxies without a $\mathrm{MBH}$ increases with decreasing halo masses at $z=0$. A larger fraction of low mass halos are devoid of central black holes for lower seed formation efficiencies. While current data in the low mass regime is still scant (Barth et al. 2005), future campaigns with the Giant Magellan Telescope or JWST are likely to probe this region of parameter space with significantly higher sensitivity.

\section{Accretion: mass growth and quasar activity}

Accretion is inevitable during the "active" phase of a galactic nucleus. Observations tell us that AGN are widespread in both the local and early Universe. All the information that we have gathered on the evolution of MBHs is indeed due to studies of AGN, as we have to await for LISA to be able to "observe" quiescent MBHs in the distant Universe. 


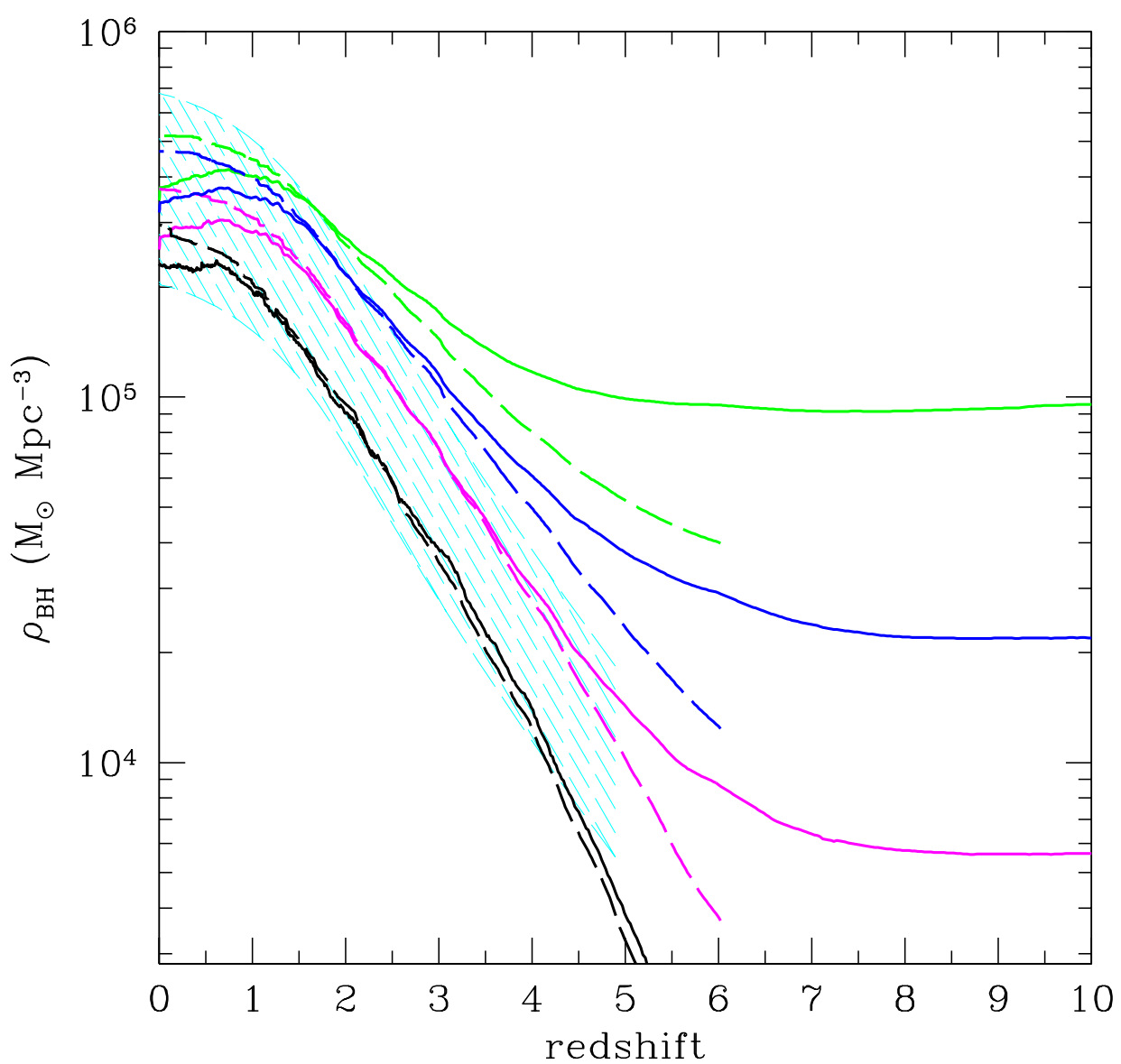

FigURE 5. Integrated black hole mass density as a function of redshift. Solid lines: total mass density locked into nuclear black holes. Dashed lines: integrated mass density accreted by black holes. Models based on BH remnants of Population III stars (lowest curve), models based on direct collapse (Lodato \& Natarajan 2006), with different efficiencies. Shaded area: constraints from Soltan-type arguments, where we have varied the radiative efficiency from a lower limit of $6 \%$ (applicable to Schwarzschild MBHs, upper envelope of the shaded area), to about $20 \%$ (Wang et al. 2006).

A key issue is then the relative importance of mergers and accretion in the build-up of the black holes, in dependence of the host properties (mass, redshift, environment).

The accretion of mass at the Eddington rate would cause a black hole mass to increase in time as

$$
M(t)=M(0) \exp \left(\frac{1-\epsilon}{\epsilon} \frac{t}{t_{\mathrm{Edd}}}\right),
$$

where $t_{\mathrm{Edd}}=0.45 \mathrm{Gyr}$ and $\epsilon$ is the radiative efficiency. The classic argument of Soltan (1982), compares the total mass of black holes today with the total radiative output by known quasars, by integration over redshift and luminosity of the luminosity function of quasars (Yu \& Tremaine 2002; Elvis et al. 2002; Marconi et al. 2004). The total energy 


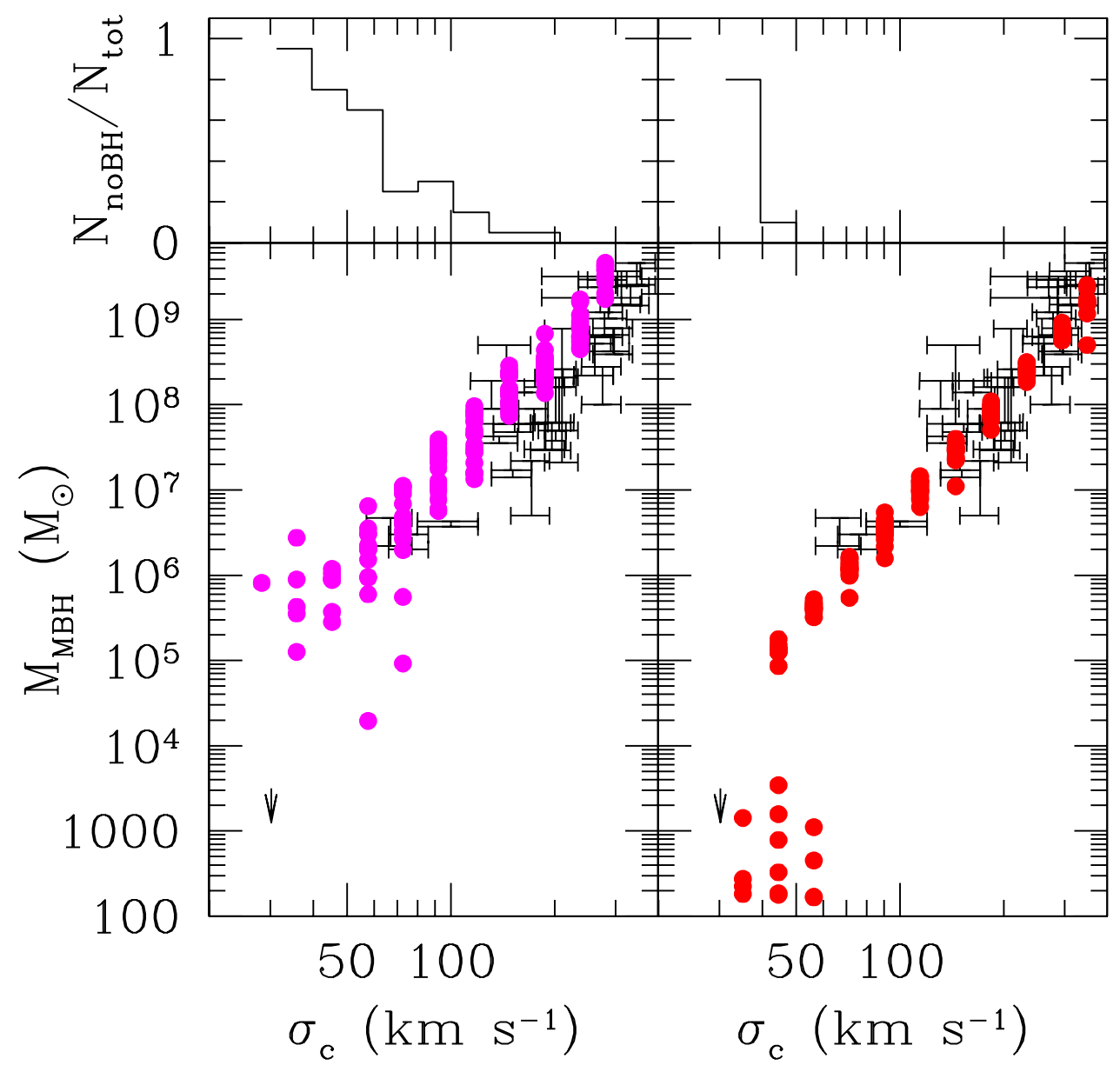

Figure 6 . The $M_{\mathrm{bh}}$-velocity dispersion $\left(\sigma_{c}\right)$ relation at $z=0$. Every circle represents the central $\mathrm{MBH}$ in a halo of given $\sigma_{c}$. Observational data are marked by their quoted errorbars, both in $\sigma_{c}$, and in $M_{\mathrm{bh}}$ (Tremaine et al. 2002). Left panel: direct collapse seeds, Population III star seeds. Top panels: fraction of galaxies at a given velocity dispersion which do not host a central MBH. Adapted from Volonteri, Lodato \& Natarajan (2007).

density can then be converted into the total mass density accreted by black holes during the active phase, by assuming a mass-to-energy conversion efficiency, $\epsilon$ (Aller \& Richstone 2002; Merloni et al. 2004; Elvis et al. 2002; Marconi et al. 2004). The similarity of the total mass in MBHs today and the total mass accreted by MBHs implies that the last $2-3$ e-folds of the mass is grown via radiatively efficient accretion, rather than accumulated through mergers or radiatively inefficient accretion. However, most ot the e-folds (corresponding to a relatively small amount of mass, say the first $10 \%$ of mass) could be gained rapidly via, e.g., radiatively inefficient accretion. This argument is particularly important at early times.

The Sloan Digital Sky survey detected luminous quasars at very high redshift, $z>6$, when the Universe was less than $1 \mathrm{Gyr}$ old. Follow-up observations confirmed that at least some of these quasars are powered by supermassive black holes with masses $\simeq 10^{9} M_{\odot}$ 


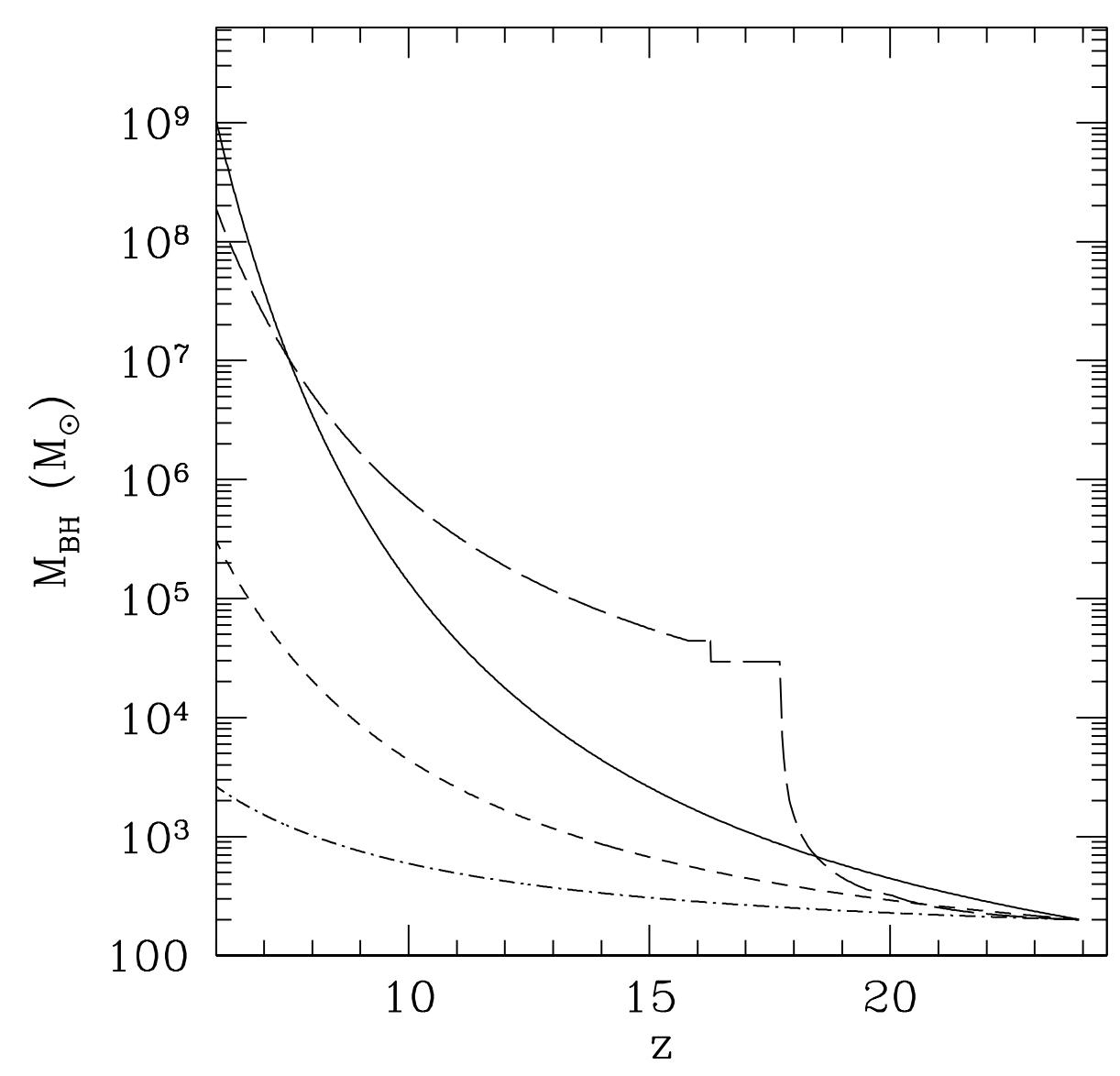

FiguRE 7. Growth of a MBH mass under different assumption for the accretion rate and efficiency. Eddington limited accretion: $\epsilon=0.1$ (solid line), $\epsilon=0.2$ (short dashed line), $\epsilon=0.4$ (dot-dashed line). Radiatively inefficient super-critical accretion, as in Volonteri \& Rees 2005 (long dashed line).

(Barth et al. 2003; Willott et al. 2005). Given a seed mass $M(0)$ at $z=50$ or less, the higher the efficiency, the longer it takes for the MBH to grow in mass by (say) 10 efoldings. If accretion is radiatively efficient, via a geometrically thin disc, the alignment of a MBH with the angular momentum of the accretion disc tends to efficiently spin holes up (see section 5), and radiative efficiencies can therefore approach 30-40\%. With such a high efficiency, $\epsilon=0.3$, it can take longer than 2 Gyr for the seeds to grow up to a billion solar masses.

Let us consider the extremely rare high redshift (say $z>15$ ) metal-free halos with virial temperatures $T_{\text {vir }}>10^{4} \mathrm{~K}$ where gas can cool even in the absence of $\mathrm{H}_{2}$ via neutral hydrogen atomic lines. The baryons can therefore collapse until angular momentum becomes important. Afterward, gas settles into a rotationally supported dense disc at the center of the halo (Mo et al. 1998; Oh \& Haiman 2002). This gas can supply fuel for accretion onto a MBH within it. Estimating the mass accreted by the MBH within 
the Bondi-Hoyle formalism, the accretion rate is initially largely above the Eddington limit (Volonteri \& Rees 2005). When the supply is super-critical the excess radiation can be trapped, as radiation pressure cannot prevent the accretion rate from being super-critical, while the emergent luminosity is still Eddington limited in case of spherical or quasi-spherical configurations (Begelman 1979; Begelman \& Meier 1982). In the spherical case, though this issue remains unclear, it still seems possible that when the inflow rate is super-critical, the radiative efficiency drops so that the hole can accept the material without greatly exceeding the Eddington luminosity. The efficiency could be low either because most radiation is trapped and advected inward, or because the flow adjusts so that the material can plunge in from an orbit with small binding energy (Abramowicz \& Lasota 1980). The creation of a radiation-driven outflow, which can possibly stop the infall of material, is also a possibility. If radiatively inefficient supercritical accretion requires metal-free conditions in exceedingly rare massive halos, rapid early growth, therefore, can happen only for a tiny fraction of $\mathrm{MBH}$ seeds. These MBHs are those powering the most luminous high redshift quasars, and later on to be found in the most biased halos today. The global MBH population, instead, evolves at a more quiet and slow pace.

\section{Probing the other hair of astrophysical black holes}

Astrophysical BHs are characterized by just 2 parameters, mass and spin, which is a measure of the angular momentum of the hole. The spin is typically expressed vis the dimensionless parameter $\hat{a} \equiv J_{h} / J_{\max }=c J_{h} / G M_{\mathrm{BH}}^{2}$, where $J_{h}$ is the angular momentum of the black hole.

The spin of a hole affects the efficiency of the "classical" accretion processes themselves; the value of $\hat{a}$ in a Kerr BH also determines how much energy is in principle extractable from the hole itself. Assuming that relativistic jets are powered by rotating black holes through the Blandford-Znajek mechanism, the so-called "spin paradigm" asserts that powerful relativistic jets are produced in AGN with fast rotating black holes (Blandford et al. 1990).

Spin-up is a natural consequence of prolonged disc-mode accretion: any hole that has (for instance) doubled its mass by capturing material with constant angular momentum axis would end up with spinning rapidly, close to the maximum allowed value Bardeen 1970; Thorne 1974). However, when an accretion disc does not lie in the equatorial plane of the $\mathrm{BH}$, that is, when the angular momentum of the accretion disc is misaligned with respect to the direction of $J_{h}$, accretion of counter-rotating material can cause the spin-down of MBHs, at least under particular conditions. A misaligned disc is subject to the Lense-Thirring precession, which tends to align the inner parts of the disc with the the angular momentum of the black hole, causing the inclination angle between the angular momentum vectors to decrease with decreasing distance from the $\mathrm{MBH}$, forcing the inner parts of the accretion disc to rotate in the equatorial plane of the MBH (Bardeen \& Petterson 1975). The orbits can be co-rotating or counter-rotating depending the value of $J_{d} / J_{h}$.

King et al. (2005) indeed argue that accretion of counter-rotating material is more common than accretion of co-rotating material. The counter-alignment condition depends on the ratio $0.5 J_{d} / J_{h}$, where $J_{h}$ and $J_{d}$ are the angular momenta of the hole and of the disc, to be compared with the cosine of the inclination angle, $\phi$. If $\cos \phi<-0.5 J_{d} / J_{h}$, the counter-alignment condition is satisfied.

However, sustained accretion from a twisted disc would align the MBH spin (and the innermost equatorial disc) with the angular momentum vector of the disc at large radii 
(Scheuer \& Feiler 1996). If the disc was initially counter-rotating with respect to the $\mathrm{MBH}$, a complete overflip would eventually occur, and then accretion of co-rotating material would act to spin up the MBH (Bardeen 1970). Early work by Moderski et al. (1998) concluded that the Bardeen-Petterson effect can be neglected because the alignment time $\left(10^{7}\right.$ years, Rees 1978) is longer than the duration of a single accretion event. Later, however, a series of papers revised the alignment timescale, suggesting that it could be much shorter (Scheuer \& Feiler 1996; Natarajan \& Pringle 1998). This framework was investigated by Volonteri et al. (2005) who argue that the lifetime of quasars is long enough that angular momentum coupling between black holes and accretion discs through the Bardeen-Petterson effect effectively forces the innermost region of accretion discs to align with black-hole spins (possibly through spin flips), and hence all AGN black-holes should have large spins (Cattaneo 2002, see also).

In this context, Volonteri et al. (2007) have explored the dependence of the alignment timescale in a Shakura \& Sunyaev (1973) disc on: viscosity $\nu$ 月f t, black hole mass $M_{\mathrm{BH}}$, Eddington ratio $f_{\text {Edd }}$, accreted mass $\Delta m$.

Within this simple picture, the timescale for disc-BH alignment can be estimated as

$$
t_{\text {align }} \simeq \frac{J_{h}}{J_{d}\left(R_{w}\right)} t_{\mathrm{acc}}\left(R_{w}\right)
$$

where $t_{\text {acc }}$ is simply the accretion timescale, $t_{\text {acc }}=R_{w}^{2} / \nu_{1}$, and $R_{w}$ marks the transition between (inner) alignment and (outer) misalignment. $R_{w}$ corresponds to the location in the disc where the timescale for radial diffusion of the warp is comparable to the local Lense-Thirring precession timescale (Scheuer \& Feiler 1996; Natarajan \& Pringle 1998). The warp radius scales with the Schwarzschild radius of the $\mathrm{BH}$ as:

$$
\frac{R_{w}}{R_{s}}=3.6 \times 10^{3} \hat{a}^{5 / 8}\left(\frac{M_{\mathrm{BH}}}{10^{8} \mathrm{M}_{\odot}}\right)^{1 / 8} f_{\mathrm{Edd}}^{-1 / 4}\left(\frac{\nu_{2}}{\nu_{1}}\right)^{-5 / 8} \alpha^{-1 / 2}
$$

Defining the mass accreted during $t_{\text {align }}$ as $m_{\text {align }}=t_{\text {align }} \dot{M}$, one gets:

$$
m_{\text {align }} \simeq M_{\mathrm{BH}} \hat{a}\left(\frac{R_{s}}{R_{w}}\right)^{1 / 2} .
$$

Therefore, for all plausible assumptions, $m_{\text {align }} \ll M_{\mathrm{BH}}$, and a series of many randomly oriented accretion events with accreted mass $\Delta m \ll m_{\text {align }}$ should result in black-hole's spin oscillating around zero. For the opposite case of $\Delta m \gg m_{\text {align }}$ the black hole will be spun-up to large positive spins; for $\Delta m \sim M_{\mathrm{BH}}$ the hole will be spun-up to $\hat{a} \sim 1$.

However, both semi-analytical models of the cosmic MBH evolution (Volonteri et al. 2005) and simulations of merger driven accretion (di Matteo et al. 2005) show that most MBHs increase their mass by an amount $\Delta m \gg m_{\text {align }}$, if the evolution of the LF of quasars is kept as a constraint. These high $\Delta m$ values are likely characteristic of the most luminous quasars and most massive black holes - especially at high redshift. We expect therefore that bright quasars at $z>3$ have large spins (upper panel in Fig. 8). High spins in bright quasars are also indicated by the high radiative efficiency of quasars,

$\dagger$ The viscosity characterizing the alignment of the disc can be different from the accretion driving viscosity, $\nu_{1}$, which is responsible for the transfer of the component of the angular momentum parallel to the spin of the disc. The relation between $\nu_{1}$ and $\nu_{2}$ is the main uncertainty of the problem, assuming of course that such two-viscosity description is adequate at all. Describing $\nu_{1}$ by the Shakura-Sunyaev parameter $\alpha$ one can show (Papaloizou \& Pringle 1983) that the regime in which $H / R<\alpha \ll 1$ ( $H$ being the disc thickness) one has $\nu_{1} / \nu_{2} \approx \alpha^{2}$. However, for high accretion rates $\alpha \ll 1$ might not be appropriate, and in such a case $\nu_{1}$ is comparable to $\nu_{2}$ (Kumar \& Pringle 1985). 
14 M. Volonteri: Evolution of massive black holes

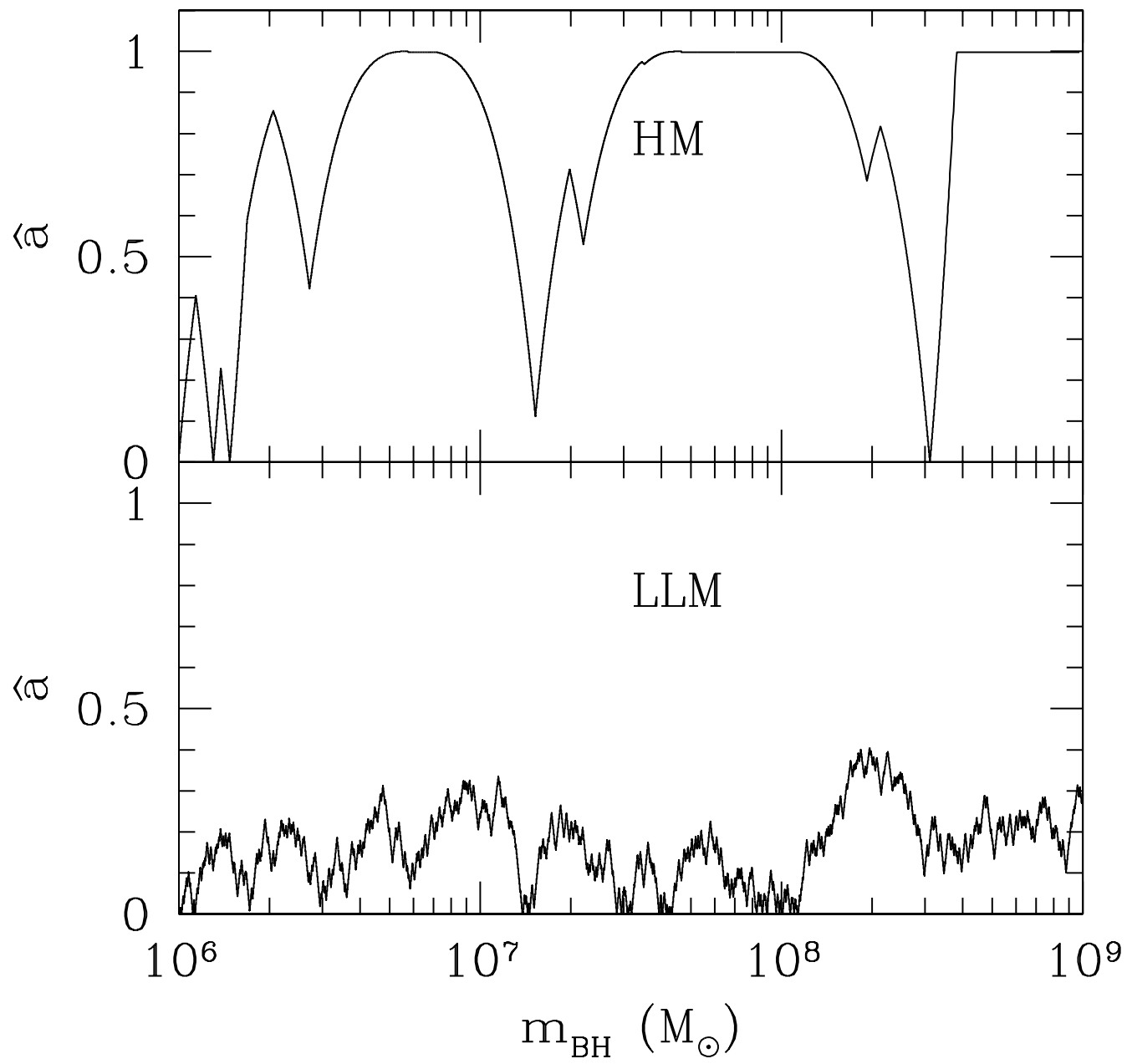

FiguRE 8. Evolution of a MBH spin during a series of accretion episodes lasting for a total of a Hubble time. Initial mass $m_{\mathrm{BH}}=10^{6} M_{\odot}$, initial spin $\hat{a}=10^{-3}$. Lower panel: the accreted mass at every accretion episode is constrained to be less than 0.01 of the MBH mass (LLM). Upper panel: the accreted mass is randomly extracted in the range 0.01-10 times the MBH mass (HM). Adapted from Volonteri, Sikora \& Lasota 2007.

as deduced from observations applying the Soltan argument (Soltan 1982; Wang et al. 2006, and references therein ).

\subsection{MBH spins and galaxy morphology}

If the events powering quasars coincide with the formation of elliptical galaxies (di Matteo et al. 2005), we might expect that the MBH hosted by an elliptical galaxy had, as last major accretion episode, a large increase in its mass. During this episode the spin increased significantly as well, possibly up to very high values.

Black holes in spiral galaxies, on the other hand, probably had their last major merger (i.e., last major accretion episode), if any, at high redshift, so that enough time elapsed for the galaxy disc to reform. Moreover, several observations suggest that single accretion events last $\simeq 10^{5}$ years in Seyfert galaxies, while the total activity lifetime (based on the fraction of disc galaxies that are Seyfert) is $10^{8}-10^{9}$ years (e.g., Kharb et al. 2006; Ho et al. 1997). This suggests that accretion events are very small and very 'compact'. 


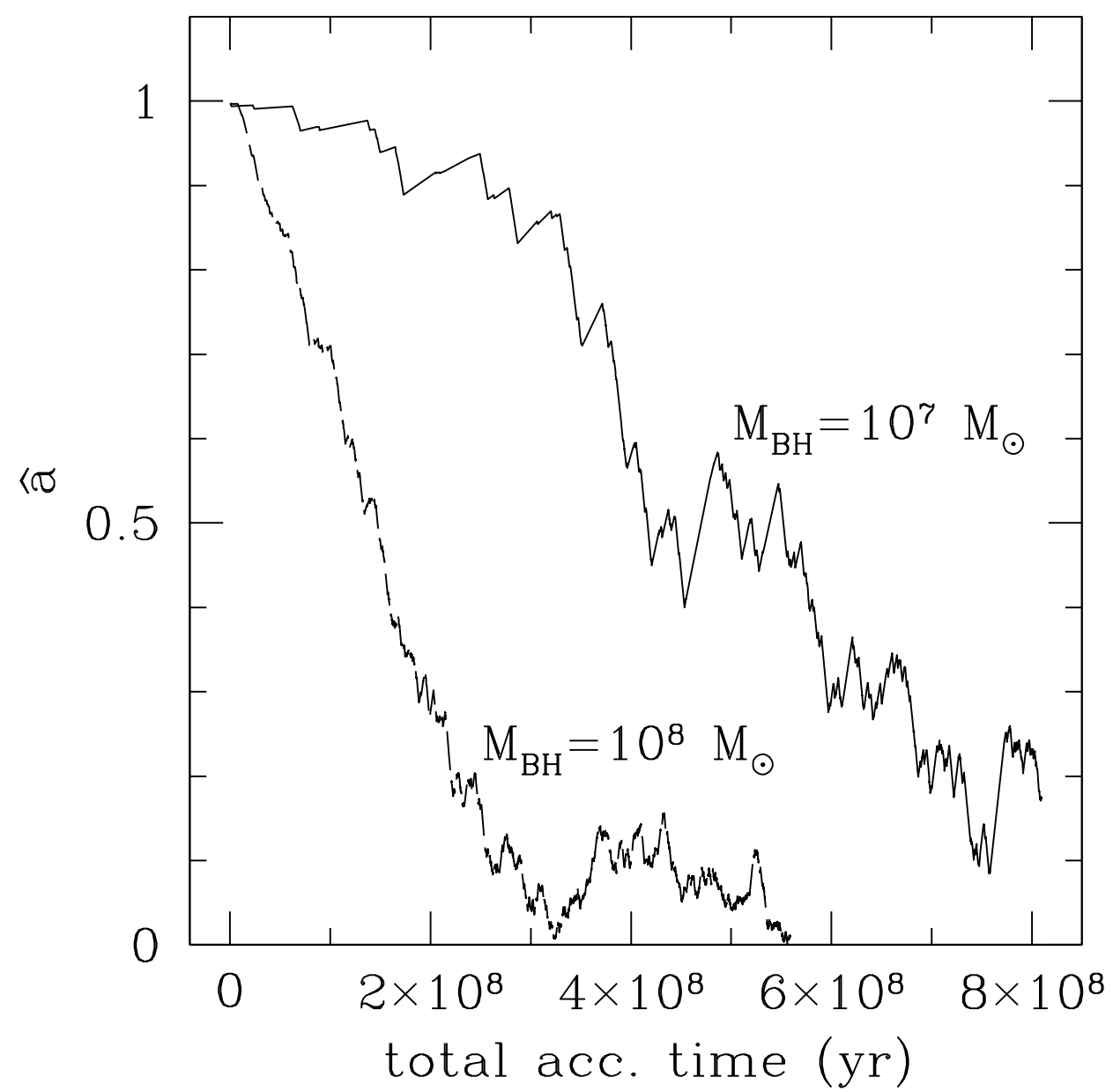

Figure 9. Evolution of MBH spins due to accretion of molecular clouds cores. We assume a lognormal distribution for the mass function of molecular clouds peaked at $\log \left(M_{M C} / \mathrm{M}_{\odot}\right)=4$, with a dispersion of 0.75 . The initial spin of the MBHs is 0.998. Upper curve: the initial $\mathrm{MBH}$ mass is $10^{7} \mathrm{M}_{\odot}$, lower curve: the initial MBH mass is $10^{8} \mathrm{M}_{\odot}$

Smaller MBHs, powering low luminosity Active Galactic Nuclei, likely grow by accreting smaller packets of material, such as tidally disrupted stars (for MBHs with mass < $2 \times 10^{6} \mathrm{M}_{\odot}$, Milosavliević et al. 2006), or possibly molecular clouds (Hopkins \& Hernquist 2006).

Compact self-gravitating cores of molecular clouds (MC) can occasionally reach subparsec regions. Although the rate of such events is uncertain, we can adopt the estimates of Kharb et al. (2006), and assume that about $10^{4}$ of such events happen. We can further assume a lognormal distribution for the mass function of $\mathrm{MC}$ close to galaxy centers (based on the Milky Way case, e.g., Perets et al. 2007). We do not distinguish here giant $\mathrm{MC}$ and clumps, and, for illustrative purpose we assume a single lognormal distribution peaked at $\log \left(M_{\mathrm{MC}} / \mathrm{M}_{\odot}\right)=4$, with a dispersion of 0.75 .

Fig. 9] shows the possible effect that accretion of molecular clouds can have on spinning 
MBHs. The result is, on the whole, similar to that produced by minor mergers of black holes (Hughes \& Blandford 2003), that is a spin down in a random walk fashion.

In a gas-poor elliptical galaxy, however, substantial populations of molecular clouds are lacking (e.g. Sage et al. 2007), eliminating this channel of MBH feeding. Main sequence stars, however, linger in galaxy centers. Tidal disruption of stars is a feeding mechanism that has been proposed long ago (Hills 1975; Rees 1988). One expects discs formed by stellar debris to form with a random orientation. Stellar disruptions would therefore contribute to the spin-down of MBHs. The number of tidal disruptions of solar type stars in an isothermal cusp per billion years can be written as:

$$
N_{*}=4 \times 10^{5}\left(\frac{\sigma}{60 \mathrm{~km} / \mathrm{s}}\right)\left(\frac{M_{\mathrm{BH}}}{10^{6} \mathrm{M}_{\odot}}\right)^{-1} .
$$

Assuming that $\mathrm{MBH}$ masses scale with the velocity dispersion, $\sigma$, of the galaxy (we adopt here the Tremaine et al. 2002 scaling), we can derive the relative mass increase for a $\mathrm{MBH}$ in 1 billion years:

$$
\frac{M_{*}}{M_{\mathrm{BH}}}=0.37\left(\frac{M_{\mathrm{BH}}}{10^{6} \mathrm{M}_{\odot}}\right)^{-9 / 8} .
$$

The maximal level of spin down would occur assuming that all the tidal disruption events form counterrotating discs, leading to retrograde accretion. Eq. 5.5 shows that a small (say $10^{6} \mathrm{M}_{\odot}$ ) $\mathrm{MBH}$ starting at $\hat{a}=0.998$ would be spun down completely, on the other hand the spin of a larger (say $10^{7} \mathrm{M}_{\odot}$ ) $\mathrm{MBH}$ would not be changed drastically. This feeding channel is likely efficient in early type discs which typically host faint bulges characterized by steep density cusps, both inside (Bahcall \& Wolf 1976) and outside (Faber et al. 1997) the sphere of influence of the BH. In this environment, the rate of stars which are tidally disrupted by MBHs (Hills 1975; Rees 1988) less massive than $10^{8} \mathrm{M} \odot$ it is non negligible (eq. 5.5, Milosavljevic et al. 2006). The situation is different for giant ellipticals: the central density profile often displays a shallow core, and tidal disruption of stars is unlikely to play a dominant role.

Summarizing, the spin of MBHs in giant elliptical galaxies is likely dominated by massive accretion events which follow galaxy mergers. Both tidal disruption of stars, and accretion of gaseous clouds is unlikely in shallow, stellar dominated galaxy cores. The spin stays consequently high. In a galaxy displaying instead power-law (cuspy) brightness profiles, the rate of stellar tidal disruptions is much higher and random small mass accretion events contribute to spin MBHs down.

The different accretion histories in elliptical and disc galaxies seem to lead to a morphology - related bimodality of black-hole spin distribution in the centers of galaxies: central black holes in giant elliptical galaxies may have (on average) much larger spins than black holes in spiral/disc galaxies.

This result is in agreement with Sikora et al. (2007) who found that disc galaxies tend to be weaker radio sources with respect to elliptical hosts. Sikora et al. (2007) therefore proposed a revised "spin paradigm", which incorporates elements of the "accretion paradigm" Ulvestad \& Ho 2001; Merloni et al. 2003; Nipoti et al. 2005; Körding et al. 2006) according to which the radio-loudness is entirely related to the states of accretion discs, similarly to radio-emission from X-ray binaries (Gallo et al. 2003), related to transitions between two different accretion modes (Livio et al. 2003). It should be emphasized that even if the production of powerful relativistic jets is conditioned by the presence of

$\dagger$ For black hole masses $\geqslant 2 \times 10^{8} \mathrm{M}_{\odot}$ the Schwarzschild radius exceeds the tidal disruption radius for main-sequence stars. 
fast rotating BHs, it also depends on the accretion rate and on the presence of disc MHD winds required to provide the initial collimation of the central Poynting flux dominated outflow.

To assess the role of spins in jet production, it is crucial to be able to measure $\mathrm{MBH}$ spins in AGN. An ingenious technique employs the $\mathrm{Fe} \mathrm{K} \alpha$ line at $6.4 \mathrm{keV}$ in the $\mathrm{X}$-ray spectrum $(2-10 \mathrm{keV})$, which is typically observed with broad asymmetric profile indicative of a relativistic disc (Miller 2007). The iron line can in principle constrain the value of black hole spins (Fabian et al. 1989; Laor 1991). The value of $\hat{a}$ affects the location of the inner radius of the accretion disc (corresponding to the innermost stable circular orbit in the standard picture), which in turn has a large impact on the shape of the line profile, because when the hole is rapidly rotating, the emission is concentrated closer in, and the line displays larger shifts. There is some evidence that this must be the case in some local Seyfert galaxies (Miniutti et al. 2004; Streblyanska et al. 2005). The assumption that the inner disc radius corresponds to the ISCO is not a trivial one, however, especially for thick discs (see Krolik 1999; Afshordi \& Paczyński 2003).

Although observations of the iron line with the Chandra and XMM X-ray satellites are extending the studies of the innermost regions of MBHs, aiming at probing black hole properties, interpretation of these studies is impeded by the inherent 'messiness' of gas dynamics. A clean probe would be a compact mass in a precessing and decaying orbit around a massive hole. The detection of gravitational waves from a stellar mass BH, or even a white dwarf, or neutron star, falling into a massive black hole (Extreme Mass Ratio Inspiral, or EMRI) can provide a unique tool to constrain the geometry of spacetime around $\mathrm{BHs}$, and as a consequence, $\mathrm{BH}$ spins. Indeed the spin is a measurable parameter, with a very high accuracy, in the gravitational waves LISA signal (Barack \& Cutler 2004; Berti et al. 2005, 2006; Lang \& Hughes 2006; Vecchio 2004). Gravitational waves from an EMRI can be used to map the spacetime of the central massive dark object. The resulting 'map' can tell us if the standard picture for the central massive object, a Kerr $\mathrm{BH}$ described by general relativity, holds.

\section{REFERENCES}

Abramowicz, M. A. \& Lasota, J. P. 1980 Spin-up of black holes by thick accretion disks. Acta Astronomica 30, 35-39.

Afshordi, N. \& Paczyński, B. 2003 Geometrically Thin Disk Accreting into a Black Hole. ApJ 592, 354-367.

Aller, M. C. \& Richstone, D. 2002 The Cosmic Density of Massive Black Holes from Galaxy Velocity Dispersions. AJ 124, 3035-3041.

Armitage, P. J. \& Natarajan, P. 2005 Eccentricity of Supermassive Black Hole Binaries Coalescing from Gas-rich Mergers. ApJ 634, 921-927.

BAHCAll, J. N. \& Wolf, R. A. 1976 Star distribution around a massive black hole in a globular cluster. ApJ 209, 214-232.

BARACK, L. \& Cutler, C. 2004 LISA capture sources: Approximate waveforms, signal-to-noise ratios, and parameter estimation accuracy. Phys. Rev. D 69 (8), 082005-+.

BArdeEn, J. M. 1970 Kerr Metric Black Holes. Nature 226, 64-+.

Bardeen, J. M. \& Petterson, J. A. 1975 The Lense-Thirring Effect and Accretion Disks around Kerr Black Holes. ApJ 195, L65+.

Barth, A. J., Greene, J. E. \& Ho, L. C. 2005 Dwarf Seyfert 1 Nuclei and the Low-Mass End of the $M_{B H}-\sigma$ Relation. ApJ 619, L151-L154.

Barth, A. J., Ho, L. C., Rutledge, R. E. \& Sargent, W. L. W. 2004 POX 52: A Dwarf Seyfert 1 Galaxy with an Intermediate-Mass Black Hole. ApJ 607, 90-102.

Barth, A. J., Martini, P., Nelson, C. H. \& Ho, L. C. 2003 Iron Emission in the z $=6.4$ Quasar SDSS J114816.64+525150.3. ApJ 594, L95-L98. 
Begelman, M. C. 1979 Can a spherically accreting black hole radiate very near the Eddington limit. MNRAS 187, 237-251.

Begelman, M. C., Blandford, R. D. \& Rees, M. J. 1980 Massive black hole binaries in active galactic nuclei. Nature 287, 307-309.

Begelman, M. C. \& Meier, D. L. 1982 Thick accretion disks - Self-similar, supercritical models. ApJ 253, 873-896.

Begelman, M. C., Volonteri, M. \& Rees, M. J. 2006 Formation of supermassive black holes by direct collapse in pre-galactic haloes. MNRAS 370, 289-298.

Berti, E., Buonanno, A. \& Will, C. M. 2005 Estimating spinning binary parameters and testing alternative theories of gravity with LISA. Phys. Rev. D $\mathbf{7 1}$ (8), 084025-+.

Berti, E., Cardoso, V. \& Will, C. M. 2006 Gravitational-wave spectroscopy of massive black holes with the space interferometer LISA. Physical Review D 73 (6), 064030-+.

Blandford, R. D., Netzer, H., Woltjer, L., Courvoisier, T. J.-L. \& Mayor, M. 1990 Active Galactic Nuclei. Saas-Fee Advanced Course 20. Lecture Notes 1990. Swiss Society for Astrophysics and Astronomy, XII, 280 pp. 97 figs.. Springer-Verlag Berlin Heidelberg New York.

Bromm, V. \& Loeb, A. 2003 Formation of the First Supermassive Black Holes. ApJ 596, $34-46$.

Bullock, J. S., Dekel, A., Kolatt, T. S., Kravtsov, A. V., Klypin, A. A., Porciani, C. \& Primack, J. R. 2001 A Universal Angular Momentum Profile for Galactic Halos. ApJ 555, 240-257.

Cattaneo, A. 2002 The growth history of supermassive black holes and the origin of the radioloud-radio-quiet dichotomy. MNRAS 333, 353-359.

Dotti, M., Colpi, M. \& HaArdt, F. 2006 Laser Interferometer Space Antenna double black holes: dynamics in gaseous nuclear discs. MNRAS 367, 103-112.

Eisenstein, D. J. \& Loeb, A. 1995 Origin of quasar progenitors from the collapse of low-spin cosmological perturbations. ApJ 443, 11-17.

Elvis, M., Risaliti, G. \& Zamorani, G. 2002 Most Supermassive Black Holes Must Be Rapidly Rotating. ApJ 565, L75-L77.

Escala, A., Larson, R. B., Coppi, P. S. \& Mardones, D. 2004 The Role of Gas in the Merging of Massive Black Holes in Galactic Nuclei. I. Black Hole Merging in a Spherical Gas Cloud. ApJ 607, 765-777.

Faber, S. M., Tremaine, S., Ajhar, E. A., Byun, Y.-I., Dressler, A., Gebhardt, K., Grillmair, C., Kormendy, J., Lauer, T. R. \& Richstone, D. 1997 The Centers of Early-Type Galaxies with HST. IV. Central Parameter Relations. AJ 114, 1771-+.

Fabian, A. C., Rees, M. J., Stella, L. \& White, N. E. 1989 X-ray fluorescence from the inner disc in Cygnus X-1. MNRAS 238, 729-736.

Fan, X., Strauss, M. A., Schneider, D. P., Gunn, J. E., Lupton, R. H., Becker, R. H., Davis, M., Newman, J. A., Richards, G. T., White, R. L., Anderson, J. E., Annis, J., Bahcall, N. A., Brunner, R. J., Csabai, I., Hennessy, G. S., Hindsley, R. B., Fukugita, M., Kunszt, P. Z., Ivezić, Ž., Knapp, G. R., McKay, T. A., Munn, J. A., Pier, J. R., Szalay, A. S. \& York, D. G. 2001 High-Redshift Quasars Found in Sloan Digital Sky Survey Commissioning Data. IV. Luminosity Function from the Fall Equatorial Stripe Sample. AJ 121, 54-65.

Ferrarese, L. \& Merritt, D. 2000 A Fundamental Relation between Supermassive Black Holes and Their Host Galaxies. ApJ 539, L9-L12.

Fryer, C. L., Woosley, S. E. \& Heger, A. 2001 Pair-Instability Supernovae, Gravity Waves, and Gamma-Ray Transients. ApJ 550, 372-382.

Gallo, E., Fender, R. P. \& Pooley, G. G. 2003 A universal radio-X-ray correlation in low/hard state black hole binaries. MNRAS 344, 60-72.

Gebhardt, K. et al. 2000 A Relationship between Nuclear Black Hole Mass and Galaxy Velocity Dispersion. ApJ 539, L13-L16.

Gould, A. \& RIx, H.-W. 2000 Binary Black Hole Mergers from Planet-like Migrations. ApJ 532, L29-L32.

Haennelt, M. G. \& Rees, M. J. 1993 The formation of nuclei in newly formed galaxies and the evolution of the quasar population. MNRAS 263, 168-178. 
Haiman, Z. 2004 Constraints from Gravitational Recoil on the Growth of Supermassive Black Holes at High Redshift. ApJ 613, 36-40.

Hills, J. G. 1975 Possible power source of Seyfert galaxies and QSOs. Nature 254, 295-298.

Ho, L. C., Filippenko, A. V. \& Sargent, W. L. W. 1997 The Influence of Bars on Nuclear Activity. ApJ 487, 591-+.

Hopkins, P. F. \& Hernquist, L. 2006 Fueling Low-Level AGN Activity through Stochastic Accretion of Cold Gas. ApJS 166, 1-36.

Hughes, S. A. \& Blandford, R. D. 2003 Black Hole Mass and Spin Coevolution by Mergers. ApJ 585, L101-L104.

Kharb, P., O’Dea, C. P., Baum, S. A., Colbert, E. J. M. \& Xu, C. 2006 A Radio Study of the Seyfert Galaxy Markarian 6: Implications for Seyfert Life Cycles. ApJ 652, 177-188.

King, A. R., Lubow, S. H., Ogilvie, G. I. \& Pringle, J. E. 2005 Aligning spinning black holes and accretion discs. MNRAS 363, 49-56.

KÖRDing, E. G., Jester, S. \& FEnder, R. 2006 Accretion states and radio loudness in active galactic nuclei: analogies with X-ray binaries. MNRAS 372, 1366-1378.

Kormendy, J. \& Gebhardt, K. 2001 Supermassive Black Holes in Nuclei of Galaxies. ArXiv Astrophysics e-prints .

Koushiappas, S. M., Bullock, J. S. \& Dekel, A. 2004 Massive black hole seeds from low angular momentum material. MNRAS 354, 292-304.

Krolik, J. H. 1999 Magnetized Accretion inside the Marginally Stable Orbit around a Black Hole. ApJ 515, L73-L76.

Kumar, S. \& Pringle, J. E. 1985 Twisted accretion discs - The Bardeen-Petterson effect. MNRAS 213, 435-442.

LANG, R. N. \& Hughes, S. A. 2006 Measuring coalescing massive binary black holes with gravitational waves: The impact of spin-induced precession. Phys. Rev. D 74 (12), 122001+ .

LAOR, A. 1991 Line profiles from a disk around a rotating black hole. ApJ 376, 90-94.

Livio, M., Pringle, J. E. \& King, A. R. 2003 The Disk-Jet Connection in Microquasars and Active Galactic Nuclei. ApJ 593, 184-188.

Lodato, G. \& Natarajan, P. 2006 Supermassive black hole formation during the assembly of pre-galactic discs. MNRAS 371, 1813-1823.

Loeb, A. \& Rasio, F. A. 1994 Collapse of primordial gas clouds and the formation of quasar black holes. ApJ 432, 52-61.

Madau, P. \& Rees, M. J. 2001 Massive Black Holes as Population III Remnants. ApJ 551, L27-L30.

Marconi, A., Risaliti, G., Gilli, R., Hunt, L. K., Maiolino, R. \& Salvati, M. 2004 Local supermassive black holes, relics of active galactic nuclei and the X-ray background. MNRAS 351, 169-185.

Mayer, L., Kazantzidis, S., Madau, P., Colpi, M., Quinn, T. \& Wadsley, J. 2006 Multiscale simulations of merging galaxies with supermassive black holes. ArXiv Astrophysics e-prints, astro-ph/0602029.

Menou, K., Haiman, Z. \& Narayanan, V. K. 2001 The Merger History of Supermassive Black Holes in Galaxies. ApJ 558, 535-542.

Merloni, A., Heinz, S. \& di Matteo, T. 2003 A Fundamental Plane of black hole activity. MNRAS 345, 1057-1076.

Merloni, A., Rudnick, G. \& Di Matteo, T. 2004 Tracing the cosmological assembly of stars and supermassive black holes in galaxies. MNRAS 354, L37-L42.

Miller, J. M. 2007 Relativistic X-ray Lines from the Inner Accretion Disks Around Black Holes. ArXiv e-prints 705.

Milosavljević, M. \& Merritt, D. 2001 Formation of Galactic Nuclei. ApJ 563, 34-62.

Milosavljević, M., Merritt, D. \& Ho, L. C. 2006 Contribution of Stellar Tidal Disruptions to the X-Ray Luminosity Function of Active Galaxies. ApJ 652, 120-125.

Miniutti, G., Fabian, A. C. \& Miller, J. M. 2004 The relativistic Fe emission line in XTE J1650-500 with BeppoSAX: evidence for black hole spin and light-bending effects? MNRAS 351, 466-472.

Mo, H. J., MAO, S. \& White, S. D. M. 1998 The formation of galactic discs. MNRAS 295, 319-336. 
Moderski, R., Sikora, M. \& Lasota, J.-P. 1998 On the spin paradigm and the radio dichotomy of quasars. MNRAS 301, 142-148.

Natarajan, P. \& Pringle, J. E. 1998 The Alignment of Disk and Black Hole Spins in Active Galactic Nuclei. ApJ 506, L97-L100.

Nipoti, C., Blundell, K. M. \& Binney, J. 2005 Radio-loud flares from microquasars and radio-loudness of quasars. MNRAS 361, 633-637.

OH, S. P. \& Haiman, Z. 2002 Second-Generation Objects in the Universe: Radiative Cooling and Collapse of Halos with Virial Temperatures above $10^{4} \mathrm{~K}$. ApJ 569, 558-572.

Papaloizou, J. C. B. \& Pringle, J. E. 1983 The time-dependence of non-planar accretion discs. MNRAS 202, 1181-1194.

Perets, H. B., Hopman, C. \& Alexander, T. 2007 Massive Perturber-driven Interactions between Stars and a Massive Black Hole. ApJ 656, 709-720.

Quinlan, G. D. 1996 The dynamical evolution of massive black hole binaries I. Hardening in a fixed stellar background. New Astronomy 1, 35-56.

REES, M. J. 1978 Emission from the nuclei of nearby galaxies - Evidence for massive black holes. In Structure and Properties of Nearby Galaxies (ed. E. M. Berkhuijsen \& R. Wielebinski), IAU Symposium, vol. 77, pp. 237-242.

ReEs, M. J. 1988 Tidal disruption of stars by black holes of 10 to the 6th-10 to the 8th solar masses in nearby galaxies. Nature 333, 523-528.

Richstone, D. et al. 1998 Supermassive black holes and the evolution of galaxies. Nature 395, $\mathrm{A} 14+$.

Salvaterra, R., HaArdt, F. \& Volonteri, M. 2007 Unresolved X-ray background: clues on galactic nuclear activity at $\mathrm{z} \& 6$. MNRAS 374, 761-768.

Scheuer, P. A. G. \& Feiler, R. 1996 The realignment of a black hole misaligned with its accretion disc. MNRAS 282, 291-+.

Schnittman, J. D. 2007 Retaining Black Holes with Very Large Recoil Velocities. ArXiv eprints $\mathbf{7 0 6 .}$

Sesana, A., HaArdt, F. \& Madau, P. $2007 a$ Interaction of Massive Black Hole Binaries with Their Stellar Environment. II. Loss Cone Depletion and Binary Orbital Decay. ApJ 660, 546-555.

Sesana, A., Volonteri, M. \& HaArdt, F. $2007 b$ The imprint of massive black hole formation models on the LISA data stream. MNRAS in press, ArXiv Astrophysics e-prints, astro-ph/0701556.

Shakura, N. I. \& Sunyaev, R. A. 1973 Black holes in binary systems. Observational appearance. A\&A 24, 337-355.

Shlosman, I., Frank, J. \& Begelman, M. C. 1989 Bars within bars - A mechanism for fuelling active galactic nuclei. Nature 338, 45-47.

Sikora, M., Stawarz, Ł. \& Lasota, J.-P. 2007 Radio Loudness of Active Galactic Nuclei: Observational Facts and Theoretical Implications. ApJ 658, 815-828.

Soltan, A. 1982 Masses of quasars. MNRAS 200, 115-122.

Spergel, D. N., Bean, R., Doré, O., Nolta, M. R., Bennett, C. L., Dunkley, J., Hinshaw, G., Jarosik, N., Komatsu, E., Page, L., Peiris, H. V., Verde, L., Halpern, M., Hill, R. S., Kogut, A., Limon, M., Meyer, S. S., Odegard, N., Tucker, G. S., Weiland, J. L., Wollack, E. \& Wright, E. L. 2007 Three-Year Wilkinson Microwave Anisotropy Probe (WMAP) Observations: Implications for Cosmology. ApJS 170, 377-408.

Streblyanska, A., Hasinger, G., Finoguenov, A., Barcons, X., Mateos, S. \& Fabian, A. C. 2005 XMM-Newton observations of the Lockman Hole. III. A relativistic Fe line in the mean X-ray spectra of type-1 and type-2 AGN. A\&A 432, 395-400.

Thorne, K. S. 1974 Disk-Accretion onto a Black Hole. II. Evolution of the Hole. ApJ 191, $507-520$.

Tremaine, S. et al. 2002 The Slope of the Black Hole Mass versus Velocity Dispersion Correlation. ApJ 574, 740-753.

Ulvestad, J. S. \& Ho, L. C. 2001 Statistical Properties of Radio Emission from the Palomar Seyfert Galaxies. ApJ 558, 561-577.

van den Bosch, F. C., Abel, T., Croft, R. A. C., Hernquist, L. \& White, S. D. M. 2002 The Angular Momentum of Gas in Protogalaxies. I. Implications for the Formation of Disk Galaxies. ApJ 576, 21-35. 
VECCHIO, A. 2004 LISA observations of rapidly spinning massive black hole binary systems. Phys. Rev. D 70 (4), 042001-+.

Volonteri, M. 2007 Gravitational Recoil: Signatures on the Massive Black Hole Population. ApJ 663, L5-L8.

Volonteri, M., HaArdt, F. \& Madau, P. 2003 The Assembly and Merging History of Supermassive Black Holes in Hierarchical Models of Galaxy Formation. ApJ 582, 559-573.

Volonteri, M., Madau, P., Quataert, E. \& Rees, M. J. 2005 The Distribution and Cosmic Evolution of Massive Black Hole Spins. ApJ 620, 69-77.

Volonteri, M. \& Rees, M. J. 2005 Rapid Growth of High-Redshift Black Holes. ApJ 633, 624-629.

Volonteri, M. \& Rees, M. J. 2006 Quasars at $z=6$ : The Survival of the Fittest. ApJ 650, $669-678$.

Volonteri, M., Sikora, M. \& Lasota, J.-P. 2007 Black-Hole Spin and Galactic Morphology. ArXiv e-prints $\mathbf{7 0 6}$.

Wang, J.-M., Chen, Y.-M., Ho, L. C. \& McLure, R. J. 2006 Evidence for Rapidly Spinning Black Holes in Quasars. ApJ 642, L111-L114.

Willott, C. J., Delfosse, X., Forveille, T., Delorme, P. \& Gwyn, S. D. J. 2005 First Results from the Canada-France High-z Quasar Survey: Constraints on the $\mathrm{z}=6$ Quasar Luminosity Function and the Quasar Contribution to Reionization. ApJ 633, 630-637.

Yu, Q. \& Tremaine, S. 2002 Observational constraints on growth of massive black holes. MNRAS 335, 965-976. 